\title{
Die Ausnutzung des Zooplanktons durch planktonfressende Fische in Abhängigkeit von abiotischen Faktoren im Oderhaff
}

\author{
KRYSTYNA WIKTOR \\ Institut für Seefischerei, Abt. Swinoujście, Polen
}

\begin{abstract}
The utilization of zooplankton by plankton-eating fishes in relation to abiotic factors in the Szczecin Gulf. The investigations were carried out in the middle area of the Szczecin Gulf, Poland, from 1955 to 1958. The seasonal and year-by-year fluctuations in the quality and quantity of zooplankton stocks may cause parallel changes in the food uptake of plankton-eating fishes. In fishes feeding primarily on zooplankton, changes were found in stomach contents, daily food consumption, efficiency of food utilization and growth rate. Fishes feeding only temporarily on zooplankton either increase their consumption of other food organisms as the zooplankton biomass decreases (2-year-old Osmerus eperlanus and Lucioperca lucioperca) or look for new feeding grounds (young of Acerina cernua and Abramis brama). In order to analyse the effects of fish predation on the zooplankton biomass, the following phenomena were investigated: (a) annual fluctuations of fish predations (mainiy O. eperlanus); (b) annual fluctuations in reproduction patterns of Cladocera species of importance as fish food (Dapbnia byalina, D. cuculata) relative to changes in the abiotic environment; (c) annual fluctuations in total mortality of the two Daphnia species mentioned. During the reproductive period the rate of reproduction in Daphnia is always higher than the maximum increase in daily food requirements of the growing O. eperlanus. Fish predation may represent a severe stress for the Daphnia populations during two periods per year. In these periods the situation is characterized by (a) decrease of prey reproduction rate and increase in daily food requirements of the growing $O$. eperlanus; (b) faster decrease of prey reproduction rate relative to a parallel decrease in food requirements of the predator.
\end{abstract}

\section{EINLEITUNG}

Das Zooplankton dient verschiedenen Fischarten als Nakrungsquelle während eines Teiles ihres Lebens oder während des gesamten Lebenszyklus. Zwischen diesen Fischarten und dem Zooplankton bilden sich bei natürlichen Gegebenheiten enge dynamische Wechselbeziehungen. Vor allem wirken dabei die zooplanktonfressenden Fische in verschiedenem Ausmaß auf die Zusammensetzung und Dichte des Zooplanktons ein.

Die in diesem Referat vorgetragenen Untersuchungsergebnisse wurden mit dem Ziel einer Analyse der Beziehungen zwischen Fisch und Plankton durchgeführt. Insbesondere ging es dabei um die Frage, wie sich die jährlichen Fluktuationen in der Zooplanktonprodukrivität aus die Fischernährung und das Gedeihen der Fische auswirken. Gleichzeitig sollte auch der Einfluß ermittelt werden, den die Fische durch ihre 
Freßtätigkeit auf die Zooplanktongemeinschaft ausüben. Es muß leider schon an dieser Stelle gesagt werden, daß die vorliegenden Ergebnisse das zweite Problem nicht zu klären vermögen. Während der in den Jahren 1955 bis 1958 durchgeführten Untersuchungen zeichneten sich aber einige Ansätze $a b$, über die ich hier berichten möchte.

\section{UNTERSUCHUNGSERGEBNISSE}

Die Forschungen wurden in den Jahren 1955 bis 1958 im mittleren Teil des Oderhaffs durchgeführt. In zweiwöchentlichen (Sommer) beziehungsweise vierwöchentlichen (Winter) Intervallen wurden qualitative Fischfänge (Tab. 1) und quantitative Planktonfänge dem Oderhaff entnommen, und zwar jeweils an den gleichen Ortlichkeiten. Die jahreszeitlichen Fluktuationen der Artzusammensetzung des Zooplanktons bewir-

Tabelle 1

Art und Zahl der untersuchten Fische in den aufgeführten Monaten. Durchschnittswerte für die Jahre 1955 bis 1958

\begin{tabular}{|c|c|c|c|c|}
\hline \multirow[b]{2}{*}{ Monat } & \multicolumn{4}{|c|}{ Fischarten } \\
\hline & $\begin{array}{c}\text { Osmerus } \\
\text { eperlanus } \\
\text { L. }\end{array}$ & $\begin{array}{l}\text { Lucioperca } \\
\text { lucioperca } \\
\text { (I.) }\end{array}$ & $\begin{array}{l}\text { Acerina } \\
\text { cernud } \\
\text { (L.) }\end{array}$ & $\begin{array}{c}\text { Abramis } \\
\text { brama } \\
\text { (L.) }\end{array}$ \\
\hline $\mathrm{I}$ & 57 & - & - & - \\
\hline II & 90 & - & - & - \\
\hline III & 55 & - & - & - \\
\hline IV & 265 & - & 3 & - \\
\hline V & 213 & 58 & - & - \\
\hline VI & 523 & 106 & 44 & - \\
\hline VII & 392 & 241 & 31 & 18 \\
\hline VIII & 350 & 431 & 87 & 93 \\
\hline $\mathrm{IX}$ & 179 & 78 & 19 & 52 \\
\hline $\mathrm{X}$ & 239 & 7 & 27 & 18 \\
\hline XI & 215 & - & - & - \\
\hline XII & 173 & 5 & 6 & - \\
\hline Zusammen: & 2751 & 926 & 217 & 181 \\
\hline
\end{tabular}

ken erhebliche Veränderungen des Nahrungsangebots für die planktonfressenden Fische. Dieser Sachverhalt kann durch die aktive Nahrungsselektion der Fische nur wenig kompensiert werden (Abb. 1). Der Unterschied zwischen der Artzusammensetzung des Zooplanktons im Milieu und im Mageninhalt ist am stärksten ausgeprägt bei der Brut und bei Jungfischstadien des Zanders (Lucioperca lucioperca [L.]), Kaulbarsches (Acerina cernua ([L.]) und Bleies (Abramis brama [L.]), am geringsten bei den Jugendstadien des Stintes (Osmerus eperlanus L.).

Die jahreszeitlichen Veränderungen in der Zooplanktonbiomasse verursachen parallele Veränderungen im Tagesquantum der Futtermenge, welche von den planktonfressenden Fischen aufgenommen wird. Dies ist aber keine durchgehende Regel; denn das Tagesquantum des Futters ist nicht nur von der Futtermenge abhängig, sondern auch von anderen Faktoren, wie z. B. der Wassertemperatur, dem Sauerstoffgehalt des 

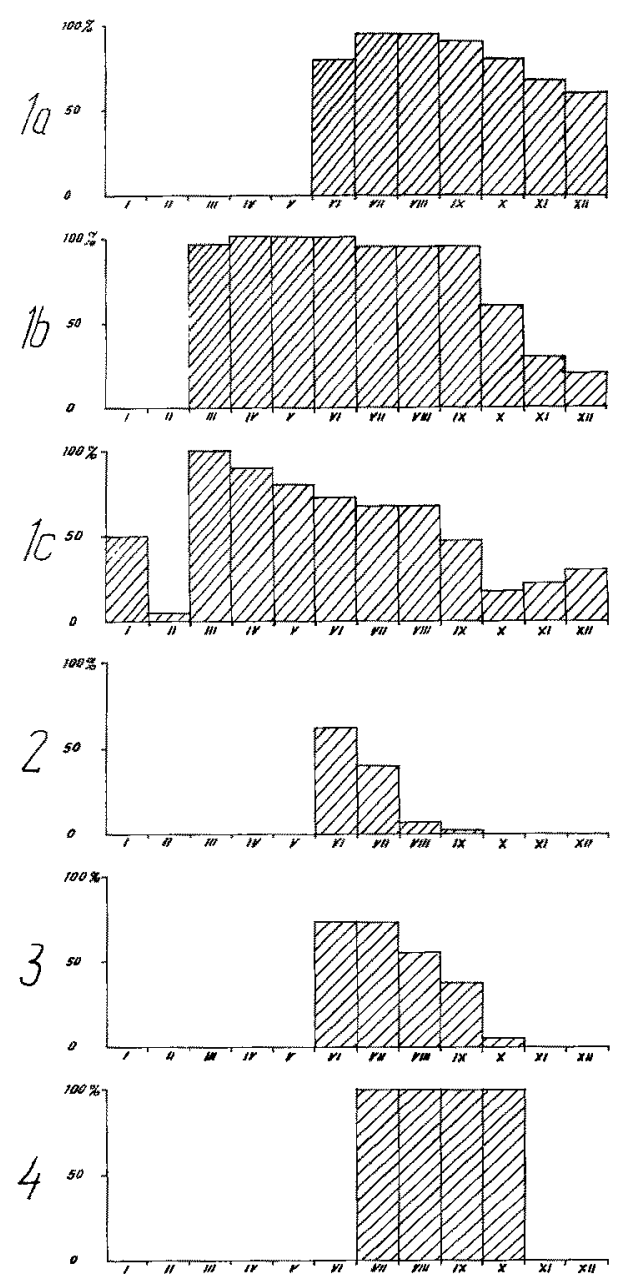

Abb. 1: Prozentualer Anteil der Zooplankter an der Gesamtmasse des Mageninhalts verschiedener Fischarten. Durchschnittswerte für die Jahre 1955 bis 1958; Waagerechte: Monate. 1: Stint; $a$ Brut, $b$ einjährig, $c$ zwei- und dreijährig; 2: Zanderbrut und Jungfische; 3 : Kaulbarsch (Jungfische); 4: Blei (Jungfische). Die Gesamtmasse der Zooplankter wurde aus der Zahl und dem durchschnittlichen Gewicht der vorhandenen Vertreter ermittelt

Wassers oder dem physiologischen Zustand des Fisches selbst (Abb. 2). Die in Abbildung 2 wiedergegebenen Veränderungen des Tageskonsums wurden an Hand des Mageninhalts am Fangtage ermittelt. Dabei wurden - unter Zugrundelegung von Angaben verschiedener Autoren über die Beziehungen zwischen Verdauungsgeschwindigkeit, Nahrungsqualität, Wassertemperatur, Fischart, Körpergröße etc. - folgende Koeffzienten (Multiplikatoren) angenommen (siehe Aufstellung Seite 451 oben).

Ganz allgemein haben die Anderungen in der Zooplanktonmenge einen beträchtlichen Einfluß auf die für die Fische zur Verfügung stehende Nahrungsqualität und -quantität und beeinflussen demzufolge auch deren Wachstumstempo. Dabei ist der 


\begin{tabular}{lccc}
\hline $\begin{array}{l}\text { Jahreszeit } \\
\text { (Monate) }\end{array}$ & $\begin{array}{c}\text { Wasser- } \\
\text { temperatur } \\
{ }^{\circ} \mathrm{C}\end{array}$ & $\begin{array}{c}\text { Art der Futterorganismen: } \\
\text { Zooplankton } \\
\text { Mageninhalt in g }\end{array}$ & $\begin{array}{c}\text { Fische } \\
\text { Mageninhalt in g }\end{array}$ \\
\hline I-III & $0-2$ & 1 & 0,5 \\
IV-V & $10-12$ & 2 & 1 \\
VI-VIII & $16-24$ & 3 & 2 \\
IX & $12-16$ & 2 & 1 \\
X & $6-10$ & 1,5 & 1 \\
XI-XII & $0-4$ & 1 & 0,5
\end{tabular}

Effekt um so stärker, je nahrungsspezifischer eine Fischart ist. Bei der Stintbrut und den einjährigen Stinten wurde im Oderhaff der Zusammenhang zwischen der Zooplanktondichte einerseits und dem Tagesquantum der aufgenommenen Nahrung sowie der Wachstumsrate andererseits recht deutlich (Tab. 2; Abb. 3). Bei den erwähnten Stintjahrgängen lassen sich auch Änderungen in der Futterverwertung deutlich nachweisen. Der Futterverwertungs-Koeffizient (Futtermenge : Gewichtszunahme in $\mathrm{g}$ Rohgewicht), welcher für die ganze Ernährungsperiode berechnet wurde, schwankte zwischen 4,1 und 4,3 in den Jahren geringer Planktonproduktivität und stieg auf 6,9 bis 10,9 in den fruchtbaren Jahren.

Die Arten, deren Nahrung nicht ausschließlich aus Zooplankton besteht, zeigen im Verlauf der quantitativen Fluktuationen des Planktons keine entsprechenden Anderungen in ihrer Wachstumsintensität. So ändern beispielsweise der dreijährige Stint

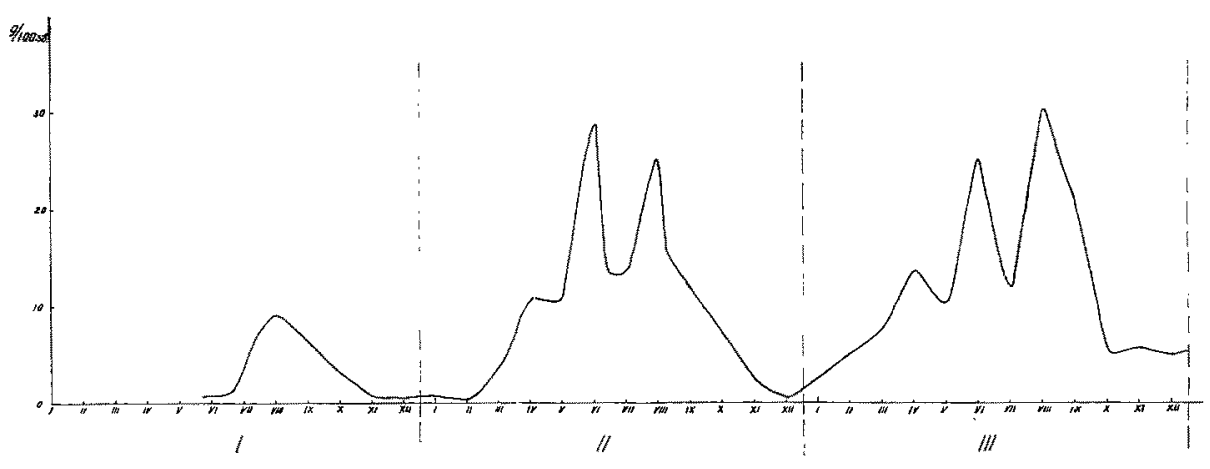

Abb. 2: Jahreszeitliche Änderungen des Tageskonsums beim Stint (Osmerus eperlanus) in den ersten 3 Lebensjahren (I, II, III). Durchschnittswerte für die Jahre 1956 bis 1958 und für jeweils 100 Fische

und der vorgestreckte Zander zu Zeiten geringer Planktondichte ihr Nahrungsobjekt; sie ernähren sich dann hauptsächlich von Stintlarven und dem Krebs Neomysis vulgaris 'Thompson. Die Jungfische von Kaulbarsch und Brassen ernährten sich nicht von Zooplankton, sondern von anderen Futterorganismen, z. B. Tendipediden-Larven, oder sie suchen sogar neue Futterplätze auf, etwa im Litoral.

Der Einfluß, welchen die planktonfressenden Fische auf die Zusammensetzung 
und Dichte des Zooplanktons ausüben, ist viel schwieriger zu umreißen. Die Zooplanktongemeinschaft wird ja immer sehr stark von physiko-chemischen, edaphischen und vielen anderen Faktoren beeinflußt. Im Oderhaff wurde bis jetzt der Einfluß der Wassertemperatur, des Salzgehaltes, der Sauerstoffkonzentration und des $\mathrm{pH}$ auf die Bildung und Entwicklung der Planktongemeinschaften untersucht und nachgewiesen (WIKTor 1957, 1959, 1961). Diese Faktoren ändern sich jahreszeitlich (WIKTor \& ZEMBRZUSKA 1959) und können zusammen mit dem Nahrungsfaktor gleichsinnig oder

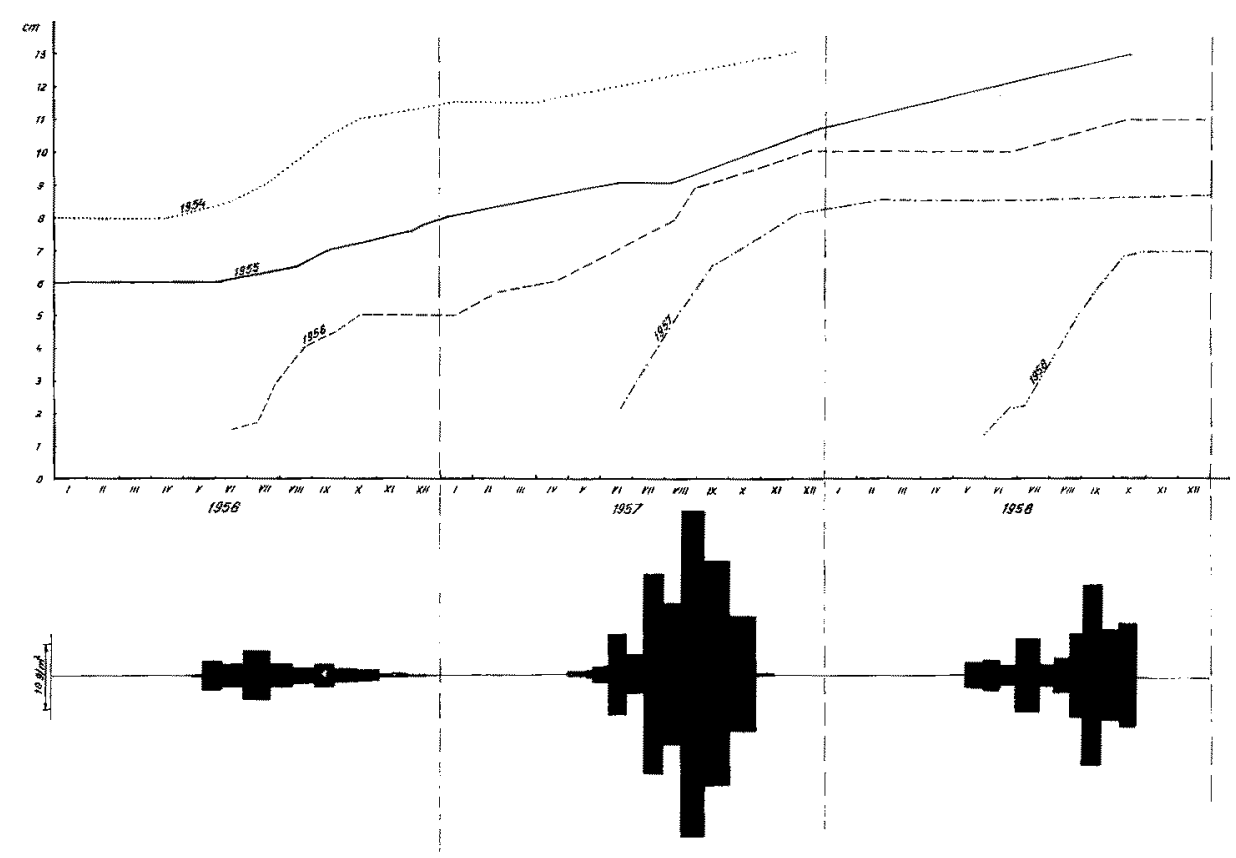

Abb. 3: Wachstumsintensität (Totallänge) verschiedener Stintgenerationen (Osmerus eperlanus) und Veränderungen in der Zooplanktonbiomasse (unten) während der Jahre 1956 bis 1958

antagonistisch auf die Planktongemeinschaft einwirken. Die physiko-chemischen und edaphischen Faktoren haben eine erhebliche Bedeutung für die Produktion der Biomasse, weil sie das Reproduktionstempo der einzelnen Planktonkomponenten steuern. Außerdem verursachen die Anderungen der Umweltfaktoren auch Veränderungen in der Sterblichkeit der Zooplankter. Es ist recht schwierig, die Verminderung der Planktonpopulation durch Fischfraß von der gesamten Sterblichkeit des Zooplanktons zu trennen.

In diesem Zusammenhang ist die Klärung der Frage wichtig, ob die Produktionsintensität des Planktons (in Abhängigkeit von physiko-chemischen und edaphischen Umweltfaktoren) und die Sterblichkeit des Planktons (u. a. in Abhängigkeit vom Fischfraß) sich parallel ändern oder antagonistisch. Zur Klärung dieser Fragen habe ich Untersuchungen über das Reproduktionstempo von Dapbnia byalina (LeYDig) und D. cuculata (G. O. SARs) durchgeführt - zwei Arten, welche als Fischnahrung im Oder- 


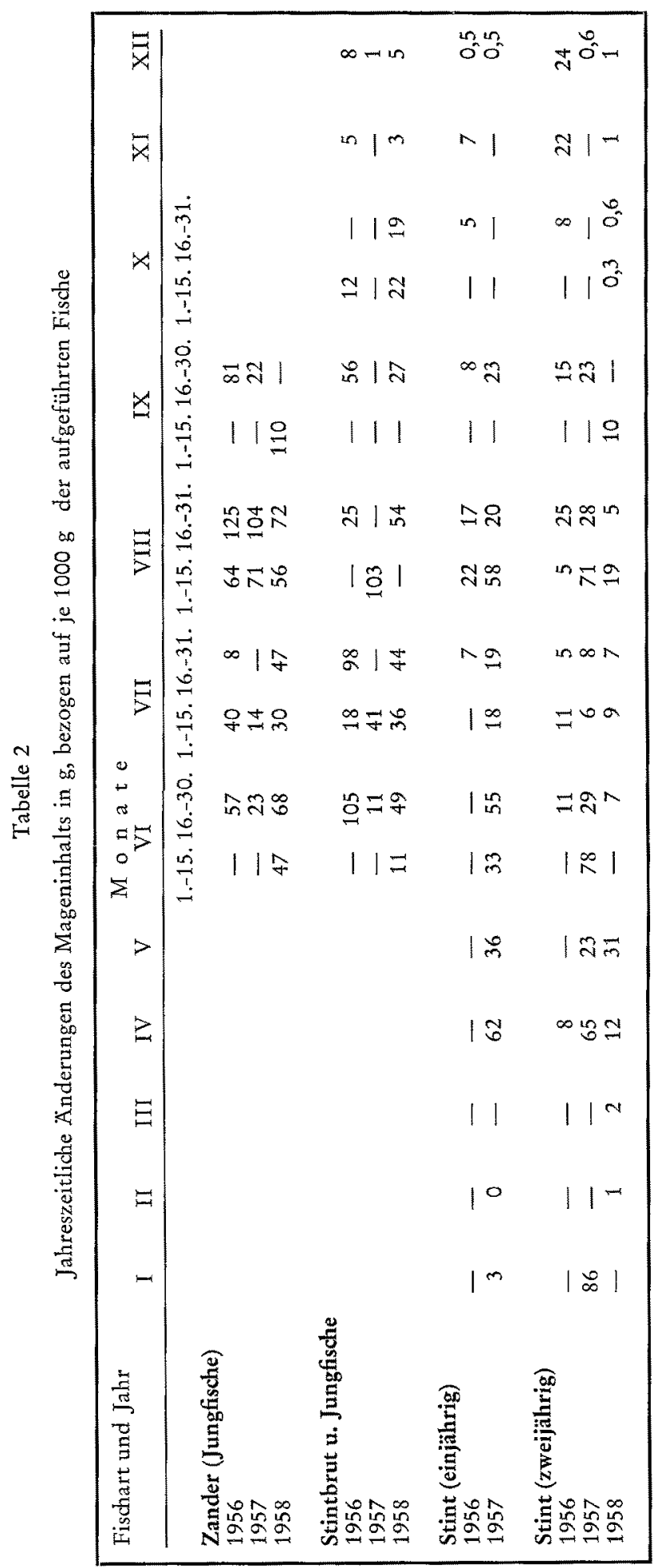


haff von großer Bedeutung sind. Die Beziehungen der jahreszeitlichen Reproduktionsschwankungen beider Arten zu den Anderungen der hydrographischen und edaphischen Verhältnisse sind sehr deutlich. Auch die Periodizität des Sterblichkeitskoeffizienten der Population beider Arten wurde untersucht (WIKTor 1961). Leider ist die Bestandsdichte der planktonfressenden Fische unbekannt. Daher ist eine Trennung der Sterblichkeit von Daphnia durch Fischfraß von der Gesamtsterblichkeit nicht möglich. Ferner unterliegt die Gesamtzehrung durch die Fischpopulationen jahreszeitlichen Veränderungen; kurz nach dem Schlüpfen und während des ersten Lebensabschnittes neugeborener Fischjahrgänge steigt sie vermutlich erheblich an.

Die bisherigen Ergebnisse erlauben den Schluß, daß die Reproduktionskapazität der Daphnia-Arten während der Fortpflanzungszeit immer größer ist als die maximale Zuwachsgeschwindigkeit des Nahrungsbedarfs der anwachsenden Stinte. Weitere Forschungen zeigten, daß im Oderhaff zwei Perioden auftreten, in denen der Fischfraß eine starke Verminderung der Daphnia-Population bewirken kann. Die erste Periode liegt in der zweiten Junihälfte und den ersten Julitagen. Sie zeichnet sich aus durch das Absinken des Reproduktionstempos der Daphnien und eine Steigerung des täglichen Futterbedarfs der Stint- und Zanderbrut. In derselben Zeit steigert sich die Gesamtsterblichkeit der Dapbnia-Populationen erheblich. Die zweite Periode liegt etwa in der $z$ weiten Septemberhälfte. Sie wird durch die klimatischen und hydrographischen Verhältnisse in den einzelnen Jahren bedingt.

$\mathrm{Zu}$ dieser Zeit vermindert sich die Reproduktionskapazität der Daphnien sehr schnell; die Fortpflanzungszeit der Daphnien nähert sich ihrem Ende. Gleichzeitig sinkt der Nahrungstagesbedarf der Stinte als Folge des Absinkens der Wassertemperatur. Dieser Vorgang geht aber langsamer vor sich als die Abnahme der Reproduktionskapazität der Daphnien. Dafür nimmt - verglichen mit den Verhältnissen im August im September die Gesamtsterblichkeit der Daphnien erheblich zu. Bosmina coregoni typica BAIRD und verschiedene Copepoden-Arten, welche im Untersuchungsgebiet leben, wurden nicht näher untersucht. Man darf aber vielleicht vermuten, daß die Auswirkung des Fischfraßes auf die Bosmina-Population einer ähnlichen Dynamik folgt, wie sie für Dapbnia nachgewiesen wurde. Bei den Copepoden dagegen gleichen sich der Verlust durch Fischfraß und der Populationszuwachs vielfach weitgehend aus.

\section{ZUSAMMENFASSUNG}

1. In den Jahren 1955-1958 wurden im Oderhaff Untersuchungen durchgeführt über die quantitative Dynamik zwischen Artzusammensetzung und Quantität des Zooplanktons einerseits und Wachstum und Nahrungsaufnahme planktonfressender Fische andererseits.

2. Die jahreszeitlich bedingten Veränderungen in der Planktonzusammensetzung üben einen starken quantitativen und qualitativen Einfluß aus auf die Nahrungszusammensetzung planktonfressender Fische (Mageninhaltsuntersuchungen).

3. Die pro Zeiteinheit aufgenommene Nahrungsmenge ist in starkem Maße von der Quantität des zur Verfügung stehenden Zooplanktons abhängig.

4. Die Dynamik der Beziehungen Fischfraß - Zooplankton und Zooplankton - Fisch- 
fraß ist eng verbunden mit jahreszeitlichen Fluktuationen der abiotischen Umweltfaktoren.

5. Futterreichtum bewirkt eine Erhöhung des Zuwachstempos und des Futterverwertungskoeffizienten der Fische.

6. Fischarten, deren Nahrung nicht ausschließlich aus Zooplankton besteht, erschließen sich in Zeiten mit geringer Zooplanktonbiomasse andere Nahrungsquellen und andere Futterplätze.

7. Der Einfluß des Fischfraßes auf die Bildung und Vermehrung der Zooplanktongemeinschaften ist schwer zu beurteilen. Er läßt sich aber nachweisen, und zwar vor allem in Perioden mit herabgesetzter Reproduktionskapazität der Planktonorganismen und mit gesteigertem Nahrungskonsum der Fische. Es wurden zwei solche Perioden pro Jahr im Oderhaff festgestellt.

\section{ZITIERTE LITERATUR}

Wiktor, K. 1957. Zooplankton Zalewu Szczecińskiego Cz. I. (Zooplankton in the Firth of Szczecin, P. I.) Prace morsk. Inst. ryback. Gdyni (oceanogr.-icbtiol.) 9, 501-547.

- 1959. Zooplankton Zalewu Szczecińskiego Cz. II. (Zooplankton in the Szczecin Firth, P. II.) Prace morsk. Inst. ryback. Gdyni (oceanogr.-icbtiol.) 10/A, 229-258.

- 1961. Wplyw warunków środowiska na zmienność populacji Bosmina coregoni, Dapbria byalina $\mathrm{i}$ Dapbnia cucullata. (The influence of habitat factors on variations in the populations of Bosmina coregoni, Daphnia byalina and Dapbnia cucullata.) Ekol. polsk. S. A. IX 5, 79-97

- \& Zembrzuska, D, 1959. Materialy do hydrografii Zalewu Szczecińskiego. (Materials for hydrography of Firth of Szczecin.) Prace morsk. Inst. ryback. Gdyni (oceanogr.-ichtiol.) 10/A, 259-269. 